\title{
I. X-RAY MINERALOGY DATA, FAR WESTERN PACIFIC, LEG 31 DEEP SEA DRILLING PROJECT ${ }^{1}$
}

\author{
H. E. Cook, I. Zemmels, and J. C. Matti, University of California, Riverside, California
}

\section{METHODS}

Semiquantitative determinations of the mineral composition of bulk samples, $2-20 \mu \mathrm{m}$, and $<2 \mu \mathrm{m}$ fractions were performed according to the methods described in the appendix of Volume 28.

The method in sample preparation, in brief, was as follows: Bulk samples were washed to remove seawater salts and ground to $<10 \mu \mathrm{m}$ under butanol. A portion of the sediment was decalcified in a sodium-acetatebuffered, acetic-acid solution ( $p \mathrm{H} \mathrm{4.5)}$. The residue was fractioned into $2-20 \mu \mathrm{m}$ and $<2 \mu \mathrm{m}$ samples by wetsieving and centrifuging. The $2-20 \mu \mathrm{m}$ samples were ground to less than $10 \mu \mathrm{m}$. These three preparations were treated with trihexylamine acetate to expand the smectites. All samples were X-rayed as random powders.

The stratigraphic positions of samples submitted for $\mathrm{X}$-ray diffraction analysis from Leg 31 are listed in Table 1. The X-ray mineralogy data are presented in Tables 2 through 16. The sample depth (in $\mathrm{m}$ ) below the sea floor in Table 1 identifies the samples as they are reported in Tables 2 through 16. No X-ray samples were submitted from Site 300 . Summary tables describing the age and lithology of the X-ray samples and listing the dominant minerals in each sample are recorded in expanded summary tables accompanying each Site Report chapter.

The amorphous content (largely consisting of biogenous silica, volcanic glass, allophane, and organic matter) was computed from the diffuse scatter of a sample. This method assumes that the diffuse scatter in excess of the diffuse scatter from the crystalline materials is a measure of the amorphous content. The diffuse scatter of the crystalline minerals is determined from the mineral calibration standards. Ideally the amorphous content varies between $0 \%$ and $100 \%$, but, in cases where the minerals in the sample have a higher degree of crystallinity than the calibration standards, negative values can result. The negative values are reported as blanks; these samples can be assumed to contain little or no amorphous material.

The crystalline minerals are quantified by the method of mutual ratios using peak heights and concentration factors derived from ratios of the diagnostic peaks of minerals with the major peak of quartz. Unquantifiable minerals, i.e., unidentified minerals and minerals for which standards are not available, are tentatively quantified using a hypothetical concentration factor of 3.0

\footnotetext{
'Institute of Geophysics and Planetary Physics, University of California, Riverside, California, Contribution No. 74-11.
}

which is applied to the major peak of the mineral. The concentrations of the quantifiable minerals is summed to $100 \%$. The amorphous content and the unquantifiable minerals are not included in the total. The unquantifiable minerals are reported on a qualitative scale as trace $(<5 \%)$, present $(5-25 \%)$, abundant $(25-65 \%)$, and major $(>65 \%)$.

The precision of the mineral determination is approximately $\pm 1 \mathrm{wt} . \%$ of the amount present. Because of differences in the crystallinity between the mineral calibration standards and the minerals in the samples, the accuracy of the reported concentrations is often less than the precision of the method allows. In terms of the reported concentration, smectites may vary by $\pm 50 \%$; micas, chlorites, cristobalite, tridymite, and goethite may vary by $\pm 20 \%$; kaolinite, amphibole, augite, the feldspars, the zeolites, palygorskite, sepiolite, and apatite may vary by $\pm 10 \%$; the minerals which have stable crystal lattices and are not members of solidsolution series or typically have limited crystal-lattice substitution in the sedimentary environment such as quartz, low-magnesium calcite, aragonite, dolomite, rhodochrosite, siderite, gibbsite, talc, barite, anatase, gypsum, anhydrite, halite, pyrite, hematite, and magnetite will vary by less than $\pm 5 \%$.

The user of the X-ray mineralogy data should bear in mind that: (1) the reported values are not absolute concentrations and that some adjustment has to be made for the amorphous content and the unquantifiable minerals; (2) in a homogeneous system of minerals, the mineral concentration trends are reliable because of the precision but when comparing mineral concentrations between different geographic regions or lithologic units additional information regarding the crystallinity of the minerals is required; and (3) the representativeness of the samples selected for X-ray diffraction analysis is the responsibility of the shipboard scientists and any questions pertaining to this aspect should be directed to them.

\section{DRILLING MUD USAGE}

Drilling mud, containing montmorillonite and barite, was used only at Site 297 between Cores 17 and 18. No $\mathrm{X}$-ray mineralogy samples were submitted from these cores.

\section{ACKNOWLEDGMENTS}

The writers wish to acknowledge the excellent work of Nicki D. Coursey in the interpretation of X-ray diffraction data, of Paul D. Johnson in X-ray data acquisition and data processing, and of Tom W. Halverson, Jr., in sample preparation. 
TABLE 1

Stratigraphic Position of X-ray Mineralogy Samples, Leg 31

\begin{tabular}{lr}
\hline \multicolumn{1}{c}{ Sample } & $\begin{array}{r}\text { Depth Below } \\
\text { (Interval in cm) }\end{array}$ \\
\hline Sloor $(\mathrm{m})$
\end{tabular}

TABLE 1 - Continued

\begin{tabular}{|c|c|}
\hline $\begin{array}{c}\text { Sample } \\
\text { (Interval in } \mathrm{cm} \text { ) }\end{array}$ & $\begin{array}{l}\text { Depth Below } \\
\text { Sea Floor (m) }\end{array}$ \\
\hline $296-26-4,74-76$ & 239.7 \\
\hline $296-30-4,74-76$ & 277.7 \\
\hline $296-31-5,15-17$ & 288.1 \\
\hline $296-34-1,100$ & 311.5 \\
\hline $296-36-2,90$ & 331.9 \\
\hline $296-40-3,44$ & 370.9 \\
\hline $296-40-3,53$ & 371.0 \\
\hline $296-40-4,16$ & 372.2 \\
\hline $296-40-4,145$ & 373.5 \\
\hline $296-41-1,12$ & 377.1 \\
\hline $296-41-2,24$ & 378.7 \\
\hline $296-41-2,93$ & 379.4 \\
\hline $296-41-3,28-30$ & 380.3 \\
\hline $296-42-1,50-52$ & 387.0 \\
\hline $296-43-1,123-125$ & 397.2 \\
\hline $296-47-1,146$ & 435.5 \\
\hline $296-52-1,127-129$ & 549.3 \\
\hline $297-3-1,109-111$ & 21.1 \\
\hline $297-4-3,79-81$ & 42.8 \\
\hline $297-5-5,41-43$ & 64.4 \\
\hline $297-6-5,79-81$ & 83.8 \\
\hline $297-12-2,66-68$ & 250.2 \\
\hline $297-14-4,72-74$ & 319.7 \\
\hline $297-24-3,76-78$ & 622.3 \\
\hline $297-26-2,133-135$ & 668.8 \\
\hline $298-2-3,120-123$ & 130.7 \\
\hline $298-5-2,135-137$ & 195.8 \\
\hline $298-6-2,95-97$ & 281.0 \\
\hline $298-9-1,131-133$ & 336.8 \\
\hline $298-11-3,14-15$ & 395.6 \\
\hline $298-14-2,106-108$ & 518.6 \\
\hline $298-15-5,80-82$ & 570.3 \\
\hline $298-16-2,130-132$ & 604.3 \\
\hline $298 \mathrm{~A}-1-1,147-149$ & 51.9 \\
\hline $299-6-5,83-85$ & 54.5 \\
\hline $299-6-5,121-123$ & 54.7 \\
\hline $299-9-5,53-55$ & 82.5 \\
\hline $299-18-4,86$ & 166.9 \\
\hline $299-26-1,99-101$ & 238.5 \\
\hline $299-33-2,88-90$ & 420.4 \\
\hline $299-38-1,112-114$ & 523.6 \\
\hline $301-2-3,74-76$ & 120.7 \\
\hline $301-4-2,80-82$ & 157.3 \\
\hline $301-4-2,120-122$ & 157.7 \\
\hline $301-8-4,100-102$ & 246.0 \\
\hline $301-13-1,132-133$ & 355.8 \\
\hline $301-15-2,142-144$ & 395.4 \\
\hline $301-17-1,83-85$ & 450.3 \\
\hline $301-18-4,117-119$ & 474.2 \\
\hline $301-18-4,136-138$ & 474.4 \\
\hline $302-2-5,130-132$ & 26.3 \\
\hline $302-3-1,71-73$ & 38.7 \\
\hline $302-5-4,147-149$ & 82.0 \\
\hline $302-7-5,86-88$ & 120.9 \\
\hline $302-10-1,63-65$ & 171.6 \\
\hline $302-15-1,86-88$ & 266.9 \\
\hline $302-17-1,14-16$ & 456.1 \\
\hline
\end{tabular}


TABLE 2

Results of X-Ray Diffraction Analysis From Hole 290

\begin{tabular}{|c|c|c|c|c|c|c|c|c|c|c|c|c|c|c|c|c|}
\hline Core & $\begin{array}{c}\text { Cored } \\
\text { Interval } \\
\text { Below Sea } \\
\text { Floor }(\mathrm{m})\end{array}$ & $\begin{array}{c}\text { Sample Depth } \\
\text { Below Sea } \\
\text { Floor (m) }\end{array}$ & हे & $\frac{\dot{U}}{\tilde{J}}$ & 䒕 & $\frac{\dot{0}}{\dot{1}}$ & $\stackrel{\varpi}{\approx}$ & $\stackrel{\text { ¿ }}{\Sigma}$ & हैं & $\frac{\dot{0}}{\Sigma}$ & . & 䄈 & 莽 & 宓 & ङัँ & வ் \\
\hline \multicolumn{17}{|c|}{ Bulk Samples } \\
\hline $\begin{array}{l}5 \\
6 \\
7 \\
8\end{array}$ & $\begin{array}{l}137.0-146.5 \\
156.0-165.5 \\
213.0-222.5 \\
241.5-251.0\end{array}$ & $\begin{array}{c}23.7 \\
74.3^{\mathrm{a}} \\
74.3^{\mathrm{b}} \\
75.7 \\
140.8 \\
159.8 \\
215.2 \\
242.3 \\
248.0\end{array}$ & $\begin{array}{l}62.8 \\
64.4 \\
48.2 \\
61.6 \\
64.7 \\
64.2 \\
68.1 \\
26.3 \\
36.0\end{array}$ & $\begin{array}{c}\overline{1.5} \\
\overline{-} \\
\overline{3} .7 \\
35.3 \\
25.6 \\
\overline{27.0}\end{array}$ & $\begin{array}{l}25.2 \\
- \\
5.3 \\
8.3 \\
1.5 \\
2.2 \\
3.2 \\
- \\
0.6\end{array}$ & $\begin{array}{l}3.6 \\
- \\
- \\
- \\
- \\
- \\
- \\
- \\
-\end{array}$ & $\begin{array}{r}18.1 \\
16.1 \\
8.2 \\
11.8 \\
21.5 \\
24.4 \\
27.1 \\
\overline{-} \\
27.8\end{array}$ & $\begin{array}{l}42.6 \\
\overline{4} \\
6.1 \\
- \\
- \\
- \\
- \\
-\end{array}$ & $\begin{array}{l}6.1 \\
- \\
- \\
4.1 \\
- \\
- \\
- \\
- \\
-\end{array}$ & $\begin{array}{r}4.5 \\
70.9 \\
8.4 \\
8.9 \\
33.4 \\
30.1 \\
31.1 \\
8.9 \\
-\end{array}$ & $\begin{array}{l}- \\
- \\
\overline{0} \\
- \\
- \\
- \\
- \\
-\end{array}$ & $\begin{array}{r}\overline{11.5} \\
73.9 \\
60.1 \\
9.9 \\
8.0 \\
11.8 \\
70.9 \\
22.8\end{array}$ & $\begin{array}{l}- \\
- \\
- \\
- \\
- \\
\overline{1.2} \\
- \\
-\end{array}$ & $\begin{array}{l}- \\
- \\
- \\
- \\
- \\
- \\
\overline{-} \\
20.2 \\
21.8\end{array}$ & $\begin{array}{c}- \\
- \\
\text { Pres } \\
\text { Pres } \\
- \\
- \\
- \\
- \\
-\end{array}$ & \\
\hline \multicolumn{17}{|c|}{$2-20 \mu \mathrm{m}$ Fraction } \\
\hline $\begin{array}{l}1 \\
2 \\
5 \\
6 \\
7\end{array}$ & $\begin{array}{c}23.0-32.5 \\
70.5-80.0 \\
137.0-146.5 \\
156.0-165.5 \\
213.0-222.5\end{array}$ & $\begin{array}{r}23.7 \\
75.7 \\
140.8 \\
159.8 \\
215.2\end{array}$ & $\begin{array}{l}41.8 \\
14.9 \\
58.2 \\
58.2 \\
58.3\end{array}$ & $\begin{array}{l}- \\
- \\
- \\
-\end{array}$ & $\begin{array}{r}33.4 \\
5.2 \\
4.2 \\
3.4 \\
3.1\end{array}$ & $\begin{array}{l}3.8 \\
- \\
- \\
-\end{array}$ & $\begin{array}{l}22.6 \\
10.0 \\
39.7 \\
42.6 \\
49.0\end{array}$ & $\begin{array}{l}33.8 \\
2.5 \\
- \\
- \\
-\end{array}$ & $\begin{array}{c}6.3 \\
0.6 \\
- \\
- \\
-\end{array}$ & $\begin{array}{l}- \\
- \\
43.1 \\
39.7 \\
28.0\end{array}$ & $\begin{array}{l}- \\
0.6 \\
- \\
- \\
-\end{array}$ & $\begin{array}{l}8 \overline{1.1} \\
13.1 \\
13.0 \\
16.7\end{array}$ & $\begin{array}{l}- \\
- \\
- \\
-\end{array}$ & $\begin{array}{l}- \\
- \\
\overline{1.3} \\
3.2\end{array}$ & $\begin{array}{l}- \\
- \\
- \\
-\end{array}$ & \\
\hline \multicolumn{17}{|c|}{$<2 \mu \mathrm{m}$ Fraction } \\
\hline $\begin{array}{l}1 \\
2 \\
5 \\
6 \\
7\end{array}$ & $\begin{array}{c}23.0-32.5 \\
70.5-80.0 \\
137.0-146.5 \\
156.0-165.5 \\
213.0-222.5\end{array}$ & $\begin{array}{r}23.7 \\
75.7 \\
140.8 \\
159.8 \\
215.2\end{array}$ & $\begin{array}{l}69.3 \\
77.8 \\
42.1 \\
47.7 \\
53.0\end{array}$ & $\begin{array}{l}- \\
- \\
- \\
-\end{array}$ & $\begin{array}{r}21.7 \\
8.9 \\
0.7 \\
0.5 \\
-\end{array}$ & $\begin{array}{l}3.4 \\
- \\
- \\
- \\
-\end{array}$ & $\begin{array}{r}13.6 \\
8.8 \\
5.5 \\
4.8 \\
8.0\end{array}$ & $\begin{array}{c}28.5 \\
9.4 \\
- \\
- \\
-\end{array}$ & $\begin{array}{l}3.7 \\
- \\
- \\
- \\
-\end{array}$ & $\begin{array}{l}23.9 \\
56.5 \\
91.6 \\
94.7 \\
87.8\end{array}$ & $\begin{array}{l}- \\
- \\
- \\
-\end{array}$ & $\begin{array}{c}\overline{16.4} \\
2.3 \\
\overline{4} .2\end{array}$ & $\begin{array}{l}- \\
\overline{-} \\
- \\
-\end{array}$ & $\begin{array}{l}- \\
- \\
- \\
-\end{array}$ & $\begin{array}{l}- \\
- \\
- \\
-\end{array}$ & $\begin{array}{c}5.1 \\
- \\
- \\
- \\
-\end{array}$ \\
\hline
\end{tabular}

${ }^{\text {a }}$ Red clay matrix.

${ }^{b}$ White crystals from red clay matrix.

TABLE 3

Results of X-Ray Diffraction Analysis From Hole 290A

\begin{tabular}{|c|c|c|c|c|c|c|c|c|c|}
\hline Core & $\begin{array}{l}\text { Cored } \\
\text { Interval } \\
\text { Below Sea } \\
\text { Floor (m) }\end{array}$ & $\begin{array}{l}\text { Sample Depth } \\
\text { Below Sea } \\
\text { Floor }(\mathrm{m})\end{array}$ & 客 & $\frac{0}{\text { गु }}$ & 崩 & $\stackrel{\Xi}{a}$ & $\frac{\dot{E}}{\dot{z}}$ & 䓃 & 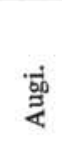 \\
\hline \multicolumn{10}{|c|}{ Bulk Samples } \\
\hline 1 & $108.5-118.0$ & 111.1 & 71.6 & 29.8 & 3.4 & 27.4 & 19.1 & 13.5 & 6.9 \\
\hline \multicolumn{10}{|c|}{$2-20 \mu \mathrm{m}$ Fraction } \\
\hline 1 & $108.5-118.0$ & 111.1 & 67.8 & & 4.7 & 38.3 & 25.6 & 21.3 & 10.1 \\
\hline \multicolumn{10}{|c|}{$<2 \mu$ m Fraction } \\
\hline 1 & $108.5-118.0$ & 111.1 & 66.8 & & & 8.5 & 68.4 & 23.1 & \\
\hline
\end{tabular}


TABLE 4

Results of X-Ray Diffraction Analysis From Hole 291

\begin{tabular}{|c|c|c|c|c|c|c|c|c|c|c|c|c|c|}
\hline Core & $\begin{array}{c}\text { Cored } \\
\text { Interval } \\
\text { Below Sea } \\
\text { Floor (m) }\end{array}$ & $\begin{array}{l}\text { Sample Depth } \\
\text { Below Sea } \\
\text { Floor }(\mathrm{m})\end{array}$ & 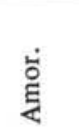 & పే & $\dot{\mathscr{b}}$ & 这 & $\frac{\text { a }}{2}$ & 茎 & 远 & हี & 总 & ฮี & 完 \\
\hline \multicolumn{14}{|c|}{ Bulk Samples } \\
\hline 1 & $0.0-3.0$ & 1.3 & 68.6 & 23.0 & - & 5.8 & 26.1 & 20.9 & 5.1 & 17.6 & - & - & 1.5 \\
\hline 2 & $60.0-69.5$ & 60.5 & 70.2 & 24.3 & - & - & 30.4 & 19.0 & 5.1 & 17.8 & - & 1.8 & 1.5 \\
\hline 3 & $79.0-88.5$ & 80.4 & 85.6 & 8.5 & - & - & 36.8 & 6.8 & - & 47.8 & - & - & - \\
\hline \multirow[t]{2}{*}{4} & $98.0-107.5$ & 102.7 & 25.0 & 5.8 & 77.0 & - & - & - & - & - & 17.2 & - & - \\
\hline & & 103.4 & 53.4 & 1.8 & 42.2 & - & 2.3 & 3.2 & - & 36.4 & 5.7 & 8.5 & - \\
\hline \multicolumn{14}{|c|}{$2-20 \mu \mathrm{m}$ Fraction } \\
\hline 1 & $0.0-3.0$ & 1.3 & 54.5 & 29.6 & - & - & 34.1 & 17.5 & 5.4 & 10.1 & - & - & 3.3 \\
\hline 2 & $60.0-69.5$ & 60.5 & 53.9 & 31.1 & - & - & 35.6 & 15.3 & 5.7 & 7.7 & - & 1.7 & 2.8 \\
\hline 3 & $79.0-88.5$ & 80.4 & 89.0 & 14.4 & - & - & 50.0 & 3.4 & 2.8 & 29.4 & - & - & - \\
\hline 4 & $98.0-107.5$ & 103.4 & 50.4 & 2.2 & 52.6 & - & 2.4 & 1.5 & - & 12.5 & 11.5 & 17.3 & - \\
\hline \multicolumn{14}{|c|}{$<2 \mu \mathrm{m}$ Fraction } \\
\hline 1 & $0.0-3.0$ & 1.3 & 71.9 & 16.0 & - & 4.8 & 13.0 & 21.2 & 11.4 & 33.6 & - & - & - \\
\hline 2 & $60.0-69.5$ & 60.5 & 68.0 & 16.3 & - & - & 12.7 & 23.3 & 8.0 & 39.6 & - & - & - \\
\hline 3 & $79.0-88.5$ & 80.4 & 78.4 & 9.5 & - & - & 16.7 & 4.9 & 2.0 & 66.8 & - & - & - \\
\hline 4 & $98.0-107.5$ & 103.4 & 60.9 & 2.2 & 20.9 & - & - & 4.1 & - & 65.7 & 5.5 & 1.6 & - \\
\hline
\end{tabular}

TABLE 5

Results of X-Ray Diffraction Analysis From Hole 291A

\begin{tabular}{|c|c|c|c|c|c|c|c|c|}
\hline Core & $\begin{array}{c}\text { Cored } \\
\text { Interval } \\
\text { Below Sea } \\
\text { Floor (m) }\end{array}$ & $\begin{array}{l}\text { Sample Depth } \\
\text { Below Sea } \\
\text { Floor }(\mathrm{m})\end{array}$ & 官 & 岂 & $\stackrel{Ð 03}{\Sigma}$ & $\stackrel{\stackrel{\text { İ }}{\Sigma}}{\Sigma}$ & $\dot{\Xi}$ & 过 \\
\hline \multicolumn{9}{|c|}{ Bulk Samples } \\
\hline 1 & $98.0-107.5$ & $\begin{array}{r}98.4 \\
106.6\end{array}$ & $\begin{array}{l}80.2 \\
88.6\end{array}$ & $\begin{array}{l}8.4 \\
8.8\end{array}$ & $\begin{array}{c}9.5 \\
-\end{array}$ & $\begin{array}{c}31.7 \\
-\end{array}$ & $\begin{array}{l}50.5 \\
91.2\end{array}$ & $\begin{array}{l}\mathrm{P} \\
\mathrm{M}\end{array}$ \\
\hline \multicolumn{9}{|c|}{$2-20 \mu \mathrm{m}$ Fraction } \\
\hline 1 & $98.0-107.5$ & $\begin{array}{r}98.4 \\
106.6\end{array}$ & $\begin{array}{l}76.5 \\
85.8\end{array}$ & $\begin{array}{l}5.2 \\
4.2\end{array}$ & $\begin{array}{c}6.5 \\
-\end{array}$ & $\begin{array}{c}18.8 \\
-\end{array}$ & $\begin{array}{l}69.5 \\
95.8\end{array}$ & $\begin{array}{l}\mathrm{P} \\
\mathrm{M}\end{array}$ \\
\hline \multicolumn{9}{|c|}{$<2 \mu \mathrm{m}$ Fraction } \\
\hline 1 & $98.0-107.5$ & $\begin{array}{r}98.4 \\
106.6\end{array}$ & $\begin{array}{l}77.5 \\
79.6\end{array}$ & $\begin{array}{l}11.7 \\
31.4\end{array}$ & $\begin{array}{l}- \\
-\end{array}$ & $\begin{array}{l}88.3 \\
68.6\end{array}$ & - & $\begin{array}{l}\mathrm{A} \\
\mathrm{M}\end{array}$ \\
\hline
\end{tabular}


TABLE 6

Results of X-Ray Diffraction Analysis From Hole 292

\begin{tabular}{|c|c|c|c|c|c|c|c|c|c|c|c|c|c|c|c|c|}
\hline Core & $\begin{array}{c}\text { Cored } \\
\text { Interval } \\
\text { Below Sea } \\
\text { Floor (m) }\end{array}$ & $\begin{array}{l}\text { Sample Depth } \\
\text { Below Sea } \\
\text { Floor }(\mathrm{m})\end{array}$ & 峁 & లే & 㫕 & $\frac{\dot{0}}{\frac{1}{4}}$ & $\frac{\text { aी }}{a}$ & 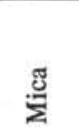 & हิ & $\stackrel{\vec{\Xi}}{\text { ¿ }}$ & 㤩 & 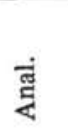 & 总 & 离 & 宊 & $\frac{\pi}{3}$ \\
\hline \multicolumn{17}{|c|}{ Bulk Samples } \\
\hline 1 & $0.0-6.5$ & 2.4 & 71.6 & 39.1 & 15.1 & - & 21.7 & 9.7 & 1.9 & 7.5 & 1.3 & & 3.8 & & - & \\
\hline 2 & $6.5-16.0$ & 8.9 & 79.4 & 32.8 & 14.4 & - & 31.0 & 12.8 & 2.9 & 6.2 & - & & - & & - & \\
\hline 3 & $16.0-25.5$ & 17.4 & 64.0 & 66.8 & 7.4 & - & 14.1 & 4.3 & 2.8 & 4.5 & - & & - & & - & \\
\hline & & 18.3 & 68.9 & 46.4 & 4.8 & - & 29.7 & 3.5 & 1.5 & 5.3 & - & & - & & 8.9 & \\
\hline 4 & $25.5-35.0$ & 30.6 & 51.5 & 82.6 & 4.4 & - & 6.0 & 1.8 & 1.7 & 2.0 & - & & 1.5 & & - & \\
\hline 5 & $35.0-44.5$ & 43.3 & 28.7 & 95.1 & 1.4 & - & 2.9 & - & 0.7 & - & - & & - & & - & \\
\hline 13 & $111.0-120.5$ & 117.2 & 37.6 & 81.1 & 1.1 & 2.3 & 3.4 & 2.5 & - & 9.7 & - & & - & & - & \\
\hline 16 & $139.5-149.0$ & 143.4 & 42.4 & 74.8 & 0.5 & - & 12.5 & 1.1 & - & 4.3 & 0.9 & & 5.9 & & - & \\
\hline 19 & $168.0-177.5$ & 174.5 & 32.5 & 92.1 & 0.5 & - & 7.4 & - & - & - & - & & - & & - & \\
\hline 35 & $320.0-329.5$ & 322.8 & 22.7 & 93.9 & 0.4 & - & 4.4 & - & - & 1.3 & - & & - & & - & \\
\hline 37 & $339.0-348.5$ & 343.2 & 80.9 & 17.9 & 11.8 & - & 56.4 & - & - & 9.3 & - & & - & & 4.7 & \\
\hline 39 & $358.0-367.5$ & 361.5 & 11.5 & 100.0 & - & - & - & - & - & - & - & & - & & - & \\
\hline \multicolumn{17}{|c|}{$2-20 \mu \mathrm{m}$ Fraction } \\
\hline 1 & $0.0-6.5$ & 2.4 & 78.0 & & 26.1 & - & 46.3 & 6.2 & 4.3 & - & 1.9 & - & - & & 15.3 & - \\
\hline 2 & $6.5-16.0$ & 8.9 & 72.6 & & 30.6 & - & 41.1 & 10.1 & 4.2 & - & - & - & 4.5 & & 9.3 & - \\
\hline 3 & $16.0-25.5$ & 17.4 & 65.8 & & 25.0 & - & 39.3 & 9.6 & 3.5 & 9.1 & 1.7 & - & 4.1 & & 7.5 & - \\
\hline & & 18.3 & 79.6 & & 14.3 & - & 53.8 & 1.8 & 2.1 & 8.2 & - & - & 2.3 & & 17.5 & - \\
\hline 4 & $25.5-35.0$ & 30.6 & 58.7 & & 29.2 & - & 39.0 & 7.7 & 5.4 & 7.6 & 3.5 & 1.1 & 6.5 & & - & $\mathbf{P}$ \\
\hline 5 & $35.0-44.5$ & 43.3 & 58.4 & & 18.6 & - & 51.8 & 4.4 & 5.4 & 9.9 & 2.6 & 0.9 & 6.4 & & - & $\mathrm{T}$ \\
\hline 13 & $111.0-120.5$ & 117.2 & 59.7 & & 9.6 & 21.4 & 22.9 & 3.6 & 3.3 & 37.2 & 2.1 & - & - & & - & - \\
\hline 16 & $139.5-149.0$ & 143.4 & 71.9 & & 5.1 & - & 49.0 & - & - & 18.2 & 4.4 & - & 23.3 & & - & - \\
\hline 19 & $168.0-177.5$ & 174.5 & 87.0 & & 9.2 & - & 72.7 & 3.2 & - & - & - & - & 1.9 & & 13.0 & - \\
\hline 35 & $320.0-329.5$ & 322.8 & 80.7 & & 12.7 & - & 62.1 & - & - & 12.3 & - & - & - & & 12.9 & - \\
\hline 37 & $339.0-348.5$ & 343.2 & 87.3 & & 8.4 & - & 74.5 & - & - & 4.8 & - & - & - & & 12.2 & - \\
\hline 39 & $358.0-367.5$ & 361.5 & 44.8 & & 6.2 & - & 30.7 & - & - & 9.8 & 53.3 & - & - & & - & - \\
\hline \multicolumn{17}{|c|}{$<2 \mu \mathrm{m}$ Fraction } \\
\hline 1 & $0.0-6.5$ & 2.4 & 69.6 & & 12.7 & & 16.3 & 20.0 & 5.8 & 42.2 & 3.0 & & & - & - & \\
\hline 2 & $6.5-16.0$ & 8.9 & 71.7 & & 15.8 & & 15.1 & 14.5 & 4.5 & 50.1 & - & & & - & - & \\
\hline \multirow[t]{2}{*}{3} & $16.0-25.5$ & 17.4 & 71.1 & & 14.2 & & 14.6 & 13.2 & 3.2 & 54.8 & - & & & - & - & \\
\hline & & 18.3 & 75.7 & & 8.7 & & 23.0 & 4.1 & 1.7 & 54.5 & - & & & - & 7.9 & \\
\hline 4 & $25.5-35.0$ & 30.6 & 68.1 & & 12.1 & & 19.0 & 11.0 & 10.0 & 44.4 & 3.5 & & & - & - & \\
\hline 5 & $35.0-44.5$ & 43.3 & 25.6 & & 0.7 & & 1.1 & - & - & 98.2 & - & & & - & - & \\
\hline 13 & $111.0-120.5$ & 117.2 & 26.1 & & 1.7 & & - & - & - & 98.3 & - & & & - & - & \\
\hline 16 & $139.5-149.0$ & 143.4 & 56.8 & & 1.2 & & 7.5 & - & - & 88.7 & 2.6 & & & - & - & \\
\hline 19 & $168.0-177.5$ & 174.5 & 78.1 & & 8.4 & & 38.2 & - & - & 39.5 & 1.4 & & & 3.9 & 8.6 & \\
\hline 35 & $320.0-329.5$ & 322.8 & 61.3 & & 3.7 & & 19.1 & - & - & 77.2 & - & & & - & - & \\
\hline 37 & $339.0-348.5$ & 343.2 & 78.5 & & 4.2 & & 39.1 & - & - & 46.5 & - & & & - & 10.2 & \\
\hline 39 & $358.0-367.5$ & 361.5 & 49.0 & & 6.4 & & 9.3 & - & - & 76.5 & 7.7 & & & - & - & \\
\hline
\end{tabular}

${ }^{\mathrm{a}} \mathrm{U}-1$ peaks at $9.46 \AA, 4.16 \AA$, and $2.705 \AA$. 
TABLE 7

Results of X-Ray Diffraction Analysis From Hole 293

\begin{tabular}{|c|c|c|c|c|c|c|c|c|c|c|c|c|c|c|c|c|}
\hline Core & $\begin{array}{c}\text { Cored } \\
\text { Interval } \\
\text { Below Sea } \\
\text { Floor }(\mathrm{m})\end{array}$ & $\begin{array}{l}\text { Sample Depth } \\
\text { Below Sea } \\
\text { Floor (m) }\end{array}$ & 岕 & نี & 㟧 & 选 & $\frac{00}{20}$ & $\frac{\Xi}{\Sigma}$ & 월 & $\stackrel{\vec{J}}{\dot{\Sigma}}$ & $\dot{\Xi}$ & 豆 & 完 & 容 & 迆 & $\frac{\pi}{5}$ \\
\hline \multicolumn{17}{|c|}{ Bulk Samples } \\
\hline 2 & $88.5-98.0$ & 91.3 & 64.5 & 1.4 & 18.5 & - & 39.4 & 14.1 & 3.6 & 20.2 & - & - & - & 2.8 & - & $\mathrm{T}$ \\
\hline 4 & $145.5-155.0$ & 147.8 & 60.6 & - & 17.8 & - & 48.8 & 15.6 & 2.8 & 9.6 & - & - & - & 5.5 & - & 1 \\
\hline 5 & $155.0-164.5$ & 160.8 & 67.3 & - & 22.5 & - & 50.5 & - & 2.2 & 12.9 & 4.1 & - & 4.2 & 3.6 & - & \\
\hline 8 & $259.5-269.0$ & 261.8 & 69.3 & - & 21.6 & - & 38.7 & 5.3 & 5.9 & 21.5 & 5.4 & - & - & 1.6 & - & \\
\hline \multirow[t]{2}{*}{9} & $269.0-278.5$ & 277.5 & 68.0 & - & 22.2 & - & 33.8 & 8.6 & 6.5 & 23.9 & 3.3 & - & - & 1.8 & - & $\mathrm{T}$ \\
\hline & & 277.6 & 67.4 & - & 22.0 & - & 50.5 & 2.6 & 2.1 & 17.3 & 4.2 & - & - & 1.3 & - & 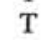 \\
\hline \multirow[t]{3}{*}{15} & $440.0-449.5$ & 440.3 & 63.8 & - & 26.5 & - & 29.2 & 21.2 & 5.5 & 17.8 & - & - & - & - & - & - \\
\hline & & 441.0 & 49.7 & - & 7.1 & - & 9.0 & 4.0 & - & 19.6 & - & 60.3 & - & - & - & - \\
\hline & & 441.3 & 66.0 & - & 20.6 & - & 32.8 & 16.6 & 3.3 & 25.4 & 1.3 & - & - & - & - & - \\
\hline 16 & $468.5-478.0$ & 472.3 & 62.6 & - & 22.8 & - & 11.6 & 22.9 & 5.7 & 36.9 & - & - & - & - & - & - \\
\hline 17 & $497.0-506.5$ & 499.3 & 52.3 & - & 4.6 & 11.8 & 2.9 & 5.2 & - & 75.5 & - & - & - & - & - & - \\
\hline 18 & $516.0-521.0$ & 516.6 & 79.6 & - & 19.8 & 28.1 & 3.2 & 22.5 & 4.9 & 21.6 & _- & - & - & _ & A & \\
\hline
\end{tabular}

2-20 $\mu \mathrm{m}$ Fraction

\begin{tabular}{|c|c|c|c|c|c|c|c|c|c|c|c|c|c|c|}
\hline 2 & $88.5-98.0$ & 91.3 & 54.9 & 23.6 & - & 42.7 & 12.5 & 4.4 & 12.0 & 2.1 & - & - & 2.6 & - \\
\hline 4 & $145.5-155.0$ & 147.8 & 47.4 & 23.8 & - & 42.3 & 14.3 & 4.0 & 10.1 & 1.5 & - & - & 4.0 & - \\
\hline 5 & $155.0-164.5$ & 160.8 & 57.5 & 21.9 & - & 44.2 & 3.8 & 2.2 & 9.2 & 2.8 & - & 11.2 & 4.6 & - \\
\hline 8 & $259.5-269.0$ & 261.8 & 84.0 & 22.6 & - & 44.2 & 6.8 & 4.3 & 16.2 & 3.2 & - & - & 2.7 & - \\
\hline \multirow[t]{2}{*}{9} & $269.0-278.5$ & 277.5 & 56.3 & 23.1 & - & 42.6 & 6.1 & 2.5 & 20.4 & 3.8 & - & - & 1.5 & - \\
\hline & & 277.6 & 56.1 & 24.6 & - & 41.1 & 9.0 & 3.2 & 17.0 & 4.0 & - & - & 1.1 & - \\
\hline \multirow[t]{3}{*}{15} & $440.0-449.5$ & 440.3 & 54.6 & 33.8 & - & 26.1 & 29.1 & 5.4 & 5.6 & - & - & - & - & - \\
\hline & & 441.0 & 40.1 & 7.6 & - & 11.5 & 5.0 & - & 9.5 & 1.0 & 65.3 & - & - & - \\
\hline & & 441.3 & 62.8 & 31.0 & - & 37.5 & 16.6 & 5.1 & 8.7 & 1.1 & - & - & - & - \\
\hline 16 & $468.5-478.0$ & 472.3 & 49.4 & 28.8 & - & 15.6 & 35.4 & 5.6 & 14.6 & - & - & - & - & - \\
\hline 17 & $497.0-506.5$ & 499.3 & 52.4 & 8.4 & 25.7 & 4.2 & 1.7 & 2.4 & 57.5 & - & - & - & - & - \\
\hline 18 & $516.0-521.0$ & 516.6 & 81.9 & 31.6 & 54.6 & 7.7 & - & 6.2 & - & - & - & - & - & A \\
\hline \multicolumn{15}{|c|}{$<2 \mu \mathrm{m}$ Fraction } \\
\hline 2 & $88.5-98.0$ & 91.3 & 59.5 & 8.6 & - & 14.4 & 11.3 & 5.1 & 58.6 & 2.1 & - & - & & - \\
\hline 4 & $145.5-155.0$ & 147.8 & 64.9 & 10.4 & - & 16.9 & 14.5 & 4.3 & 52.4 & 1.4 & - & - & & - \\
\hline 5 & $155.0-164.5$ & 160.8 & 65.2 & 6.7 & - & 15.3 & 4.7 & 3.9 & 65.9 & 1.4 & - & 2.1 & & - \\
\hline 8 & $259.5-269.0$ & 261.8 & 60.0 & 6.5 & - & 7.6 & 3.6 & 3.0 & 77.0 & 2.3 & - & - & & - \\
\hline \multirow[t]{2}{*}{9} & $269.0-278.5$ & 277.5 & 54.3 & 7.0 & - & 9.3 & 10.8 & 4.5 & 66.6 & 1.8 & - & - & & - \\
\hline & & 277.6 & 56.6 & 4.2 & - & 10.5 & - & 3.2 & 78.1 & 3.9 & - & - & & - \\
\hline \multirow[t]{3}{*}{15} & $440.0-449.5$ & 440.3 & 56.9 & 11.0 & - & 4.0 & 17.9 & 5.0 & 62.1 & - & - & - & & - \\
\hline & & 441.0 & 58.5 & 2.8 & - & 5.9 & - & - & 84.1 & - & 7.2 & - & & - \\
\hline & & 441.3 & 60.9 & 9.8 & - & 8.0 & 10.6 & 3.7 & 68.0 & - & - & - & & - \\
\hline 16 & $468.5-478.0$ & 472.3 & 44.6 & 8.1 & - & 2.7 & 11.5 & 3.8 & 74.0 & - & - & - & & - \\
\hline 17 & $497.0-506.5$ & 499.3 & 25.0 & 0.4 & 1.8 & - & - & - & 97.8 & - & - & - & & - \\
\hline 18 & $516.0-521.0$ & 516.6 & 77.3 & 14.5 & 11.5 & 2.5 & 9.4 & 3.3 & 58.7 & - & - & - & & A \\
\hline
\end{tabular}

${ }^{\mathrm{a}} \mathrm{U}-1$ peaks at $9.46 \AA, 4.16 \AA$, and $2.705 \AA$. 
TABLE 8

Results of X-Ray Diffraction Analysis From Hole 294

\begin{tabular}{|c|c|c|c|c|c|c|c|c|c|c|c|c|}
\hline Core & $\begin{array}{c}\text { Cored } \\
\text { Interval } \\
\text { Below Sea } \\
\text { Floor (m) }\end{array}$ & $\begin{array}{l}\text { Sample Depth } \\
\text { Below Sea } \\
\text { Floor (m) }\end{array}$ & 宫 & 崩 & 这 & $\frac{m}{a}$ & ¿ூّ & 을 & $\stackrel{\vec{\Xi}}{\Sigma}$ & 苞 & 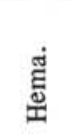 & 过 \\
\hline \multicolumn{13}{|c|}{ Bulk Samples } \\
\hline 1 & $0.0-7.5$ & 5.3 & 60.8 & 34.2 & - & 18.6 & 38.6 & 5.2 & 3.4 & - & - & - \\
\hline 3 & $74.0-83.5$ & 80.9 & 64.5 & 9.4 & 26.0 & - & - & - & 64.6 & - & - & - \\
\hline \multirow[t]{2}{*}{4} & $93.0-102.5$ & 96.8 & 81.2 & 21.1 & 41.1 & - & 9.9 & - & 24.7 & 3.1 & - & M \\
\hline & & 99.0 & 76.9 & 5.9 & 24.8 & 7.1 & - & - & 28.1 & - & 34.2 & $\mathbf{P}$ \\
\hline 6 & $105.5-112.0$ & 106.9 & 4.7 & 1.3 & - & - & - & - & 28.9 & - & 69.8 & - \\
\hline \multicolumn{13}{|c|}{$2-20 \mu \mathrm{m}$ Fraction } \\
\hline 1 & $0.0-7.5$ & 5.3 & 49.3 & 39.8 & - & 23.4 & 31.8 & 5.0 & - & - & - & - \\
\hline 3 & $74.0-83.5$ & 80.9 & 48.0 & 7.6 & 17.2 & - & 10.4 & 1.3 & 63.4 & - & - & - \\
\hline \multirow[t]{2}{*}{4} & $93.0-102.5$ & 96.8 & 78.2 & 14.9 & 40.4 & - & 12.4 & - & 24.1 & 8.2 & - & A \\
\hline & & 99.0 & 76.0 & 7.7 & 40.2 & 8.1 & 3.9 & - & 29.3 & - & 10.7 & P \\
\hline 6 & $105.5-112.0$ & 106.9 & 51.3 & 11.8 & 17.5 & 3.6 & 5.0 & 3.9 & 25.1 & - & 33.1 & - \\
\hline \multicolumn{13}{|c|}{$<2 \mu \mathrm{m}$ Fraction } \\
\hline 1 & $0.0-7.5$ & 5.3 & 70.8 & 28.4 & - & 15.9 & 38.1 & 4.8 & 12.9 & - & - & - \\
\hline 3 & $74.0-83.5$ & 80.9 & 58.6 & 3.2 & 10.8 & - & - & - & 86.0 & - & - & - \\
\hline \multirow[t]{2}{*}{4} & $93.0-102.5$ & 96.8 & 83.1 & 17.2 & 33.6 & - & 14.1 & - & 35.2 & - & - & M \\
\hline & & 99.0 & 80.2 & 3.3 & 8.9 & - & - & - & 32.4 & - & 55.3 & $P$ \\
\hline 6 & $105.5-112.0$ & 106.9 & 16.0 & 0.7 & - & - & - & - & 28.9 & - & 70.5 & - \\
\hline
\end{tabular}

TABLE 9

Results of X-Ray Diffraction Analysis From Hole 295

\begin{tabular}{|c|c|c|c|c|c|c|c|c|c|c|c|}
\hline Core & $\begin{array}{c}\text { Cored } \\
\text { Interval } \\
\text { Below Sea } \\
\text { Floor (m) }\end{array}$ & $\begin{array}{l}\text { Sample Depth } \\
\text { Below Sea } \\
\text { Floor (m) }\end{array}$ & 完 & 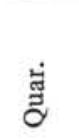 & 这 & 檪 & $\stackrel{\text { อี }}{\Sigma}$ & 을 & $\dot{\tilde{\Xi}}$ & હ் & ¿் \\
\hline \multicolumn{12}{|c|}{ Bulk Samples } \\
\hline 1 & $101.0-110.5$ & 107.8 & 66.0 & 27.4 & 13.4 & 7.2 & 18.2 & 3.4 & 30.5 & - & - \\
\hline 2 & $120.0-129.5$ & 121.9 & 70.2 & 12.2 & 29.7 & 11.7 & 7.1 & - & $\overline{0}$ & 39.3 & $\overline{\mathrm{s}}$ \\
\hline 3 & $139.0-148.5$ & $\begin{array}{l}128.6 \\
143.9\end{array}$ & $\begin{array}{l}79.5 \\
80.9\end{array}$ & $\begin{array}{r}5.5 \\
10.3\end{array}$ & $\begin{array}{l}25.4 \\
14.4\end{array}$ & $\begin{array}{l}3.7 \\
7.5\end{array}$ & - & - & $\begin{array}{l}10.7 \\
12.1\end{array}$ & $\begin{array}{l}54.7 \\
55.8\end{array}$ & $\begin{array}{l}\mathrm{M} \\
\mathrm{M}\end{array}$ \\
\hline \multicolumn{12}{|c|}{$2-20 \mu$ m Fraction } \\
\hline 1 & $101.0-110.5$ & 107.8 & 57.5 & 35.5 & 17.0 & 12.0 & 23.7 & 3.3 & 8.5 & - & - \\
\hline 2 & $120.0-129.5$ & 121.9 & 48.3 & 8.0 & 16.8 & 4.0 & 2.8 & - & 19.6 & 48.9 & - \\
\hline & & 128.6 & 64.7 & 3.5 & 19.0 & 4.9 & 2.8 & - & 6.6 & 63.2 & $\mathbf{P}$ \\
\hline 3 & $129.0-148.5$ & 143.9 & 69.5 & 11.6 & 9.4 & 5.2 & - & - & 6.6 & 67.3 & $P$ \\
\hline \multicolumn{12}{|c|}{$<2 \mu$ m Fraction } \\
\hline 1 & $101.0-110.5$ & 107.8 & 59.9 & 17.7 & 7.0 & 6.4 & 14.0 & 4.3 & 50.6 & - & - \\
\hline 2 & $120.0-129.5$ & 121.9 & 60.1 & 5.4 & 14.9 & - & - & - & 78.0 & 1.7 & - \\
\hline & & 128.6 & 82.9 & 17.5 & - & - & - & - & 71.4 & 11.1 & $\mathrm{M}$ \\
\hline 3 & $139.0-148.5$ & 143.9 & 82.8 & 22.6 & - & - & - & - & 64.3 & 13.0 & M \\
\hline
\end{tabular}


TABLE 10

Results of X-Ray Diffraction Analysis From Hole 295

\begin{tabular}{|c|c|c|c|c|c|c|c|c|c|c|c|c|}
\hline Core & $\begin{array}{c}\text { Cored } \\
\text { Interval } \\
\text { Below Sea } \\
\text { Floor }(\mathrm{m})\end{array}$ & $\begin{array}{l}\text { Sample Depth } \\
\text { Below Sea } \\
\text { Floor }(\mathrm{m})\end{array}$ & 完 & गี & 岕 & $\stackrel{\square}{\frac{\pi}{2}}$ & $\begin{array}{l}\dot{0} \\
\ddot{g}\end{array}$ & $\stackrel{\text { J }}{\Sigma}$ & 己่ & 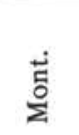 & 苞 & $\sum^{30}$ \\
\hline \multicolumn{13}{|c|}{ Bulk Samples } \\
\hline 1 & $0.0-6.5$ & 2.5 & 59.7 & 46.1 & 22.3 & 10.9 & & 17.5 & 3.1 & - & & - \\
\hline 2 & $6.5-16.0$ & 14.2 & 42.6 & 64.4 & 13.1 & 6.8 & & 12.8 & 1.6 & 1.3 & & - \\
\hline 3 & $16.0-25.5$ & 19.2 & 48.3 & 72.5 & 9.3 & 4.6 & & 7.0 & 1.8 & 4.8 & & - \\
\hline 9 & $73.0-82.5$ & 78.9 & 43.9 & 63.7 & 15.0 & 6.3 & & 12.7 & 2.3 & - & & - \\
\hline 19 & $168.0-177.5$ & 170.3 & 38.8 & 82.0 & 7.2 & 3.3 & & 7.5 & - & - & & - \\
\hline 22 & $196.5-206.0$ & 198.8 & 33.1 & 87.7 & 5.4 & 2.2 & & 4.7 & - & - & & - \\
\hline 23 & $206.0-215.5$ & 208.7 & 40.4 & 80.7 & 7.9 & 4.3 & & 7.1 & - & - & & - \\
\hline 26 & $234.5-244.0$ & 239.7 & 50.0 & 73.7 & 10.1 & 7.0 & & 7.6 & - & 1.5 & & - \\
\hline 30 & $272.5-282.0$ & 277.7 & 41.5 & 91.6 & 2.9 & 1.4 & & 3.0 & - & 1.1 & & - \\
\hline 31 & $282.0-291.5$ & 288.1 & 57.7 & 75.8 & 6.2 & 9.0 & & 6.3 & - & 2.8 & & - \\
\hline 34 & $310.5-320.0$ & 311.5 & 47.4 & 90.5 & 3.4 & 2.2 & & 2.4 & - & 1.5 & & - \\
\hline 36 & $329.5-339.0$ & 331.9 & 38.3 & 92.7 & 1.5 & 1.9 & & 2.3 & - & 1.7 & & - \\
\hline \multirow[t]{4}{*}{40} & $367.5-377.0$ & 370.9 & 78.4 & 9.2 & 3.3 & 50.2 & & 4.1 & - & 21.9 & & 11.3 \\
\hline & & 371.0 & 71.0 & 14.1 & 4.9 & 58.1 & & - & - & 2.2 & & 20.7 \\
\hline & & 372.2 & 77.7 & 3.9 & 12.5 & 56.3 & & - & 1.3 & 13.4 & & 12.5 \\
\hline & & 373.5 & 52.8 & 45.7 & 2.1 & 36.1 & & - & - & 5.1 & & 11.1 \\
\hline \multirow[t]{4}{*}{41} & $377.0-386.5$ & 377.1 & 62.1 & 5.3 & 5.1 & 63.8 & & - & - & 9.4 & & 16.4 \\
\hline & & 378.7 & 67.4 & 21.9 & 5.3 & 39.4 & & - & 1.8 & 25.0 & & 6.6 \\
\hline & & 379.4 & 74.8 & 4.2 & 4.6 & 66.4 & & - & - & 7.4 & & 17.4 \\
\hline & & 380.3 & 73.2 & - & 5.0 & 60.9 & & - & - & 20.0 & & 14.1 \\
\hline 42 & $386.5-396.0$ & 387.0 & 78.6 & 16.4 & 3.7 & 48.9 & & - & - & 11.0 & & 19.9 \\
\hline 43 & $396.0-405.5$ & 397.2 & 77.0 & 25.4 & 3.8 & 49.4 & & - & - & 5.3 & & 16.1 \\
\hline 47 & $434.0-443.5$ & 435.5 & 92.1 & 48.7 & 3.7 & 43.5 & & - & - & 4.1 & & - \\
\hline 52 & $548.0-557.5$ & 549.3 & 66.8 & 23.9 & 3.5 & 57.4 & & - & - & 2.3 & & 12.9 \\
\hline \multicolumn{13}{|c|}{$2-20 \mu \mathrm{m}$ Fraction } \\
\hline 1 & $0.0-6.5$ & 2.5 & 59.1 & & 49.4 & 24.6 & & 22.1 & 3.9 & - & - & - \\
\hline 2 & $6.5-16.0$ & 14.2 & 52.5 & & 54.0 & 24.7 & & 17.6 & 3.7 & - & - & - \\
\hline 3 & $16.0-25.5$ & 19.2 & 57.6 & & 42.7 & 21.7 & & 23.4 & 5.1 & 7.1 & - & - \\
\hline 9 & $73.0-82.5$ & 78.9 & 52.8 & & 48.6 & 21.2 & & 25.7 & 4.5 & - & - & - \\
\hline 19 & $168.0-177.5$ & 170.3 & 51.4 & & 48.1 & 24.1 & & 24.2 & 3.6 & - & - & - \\
\hline 22 & $196.5-206.0$ & 198.8 & 49.6 & & 47.5 & 23.8 & & 24.6 & 4.1 & - & - & - \\
\hline 23 & $206.0-215.5$ & 208.7 & 66.6 & & 51.3 & 26.5 & & 17.8 & 4.4 & - & - & - \\
\hline 26 & $234.5-244.0$ & 239.7 & 66.9 & & 49.1 & 30.7 & & 17.8 & 2.4 & - & - & - \\
\hline 30 & $272.5-282.0$ & 277.7 & 82.1 & & 40.4 & 29.2 & & 24.3 & 6.1 & - & - & - \\
\hline 31 & $282.0-291.5$ & 288.1 & 82.1 & & 36.8 & 41.8 & & 19.4 & 2.0 & - & - & - \\
\hline 34 & $310.5-320.0$ & 311.5 & 86.4 & & 41.0 & 36.9 & & 20.0 & 2.2 & - & - & - \\
\hline 36 & $329.5-339.0$ & 331.9 & 70.1 & & 30.9 & 32.7 & & 14.2 & 2.6 & 12.0 & - & 7.5 \\
\hline \multirow{4}{*}{40} & $367.5-377.0$ & 370.9 & 80.2 & & 7.0 & 68.5 & & - & - & - & - & 24.5 \\
\hline & & 371.0 & 71.6 & & 4.8 & 67.7 & & - & 5.4 & - & - & 22.0 \\
\hline & & 372.2 & 74.1 & & 11.1 & 72.2 & & - & 2.9 & - & - & 13.9 \\
\hline & & 373.5 & 67.4 & & 6.5 & 75.7 & & - & - & - & - & 17.8 \\
\hline \multirow[t]{4}{*}{41} & $377.0-386.5$ & 377.1 & 64.9 & & 5.9 & 78.4 & & - & - & - & - & 15.6 \\
\hline & & 378.7 & 70.9 & & 7.7 & 69.0 & & - & 1.5 & 11.7 & 1.0 & 9.1 \\
\hline & & 379.4 & 79.9 & & 6.9 & 78.9 & & - & - & - & - & 14.2 \\
\hline & & 380.3 & 79.6 & & 7.1 & 77.1 & & - & - & - & - & 15.8 \\
\hline 42 & $386.5-396.0$ & 387.0 & 81.4 & & 5.8 & 68.8 & & - & - & 3.0 & - & 22.4 \\
\hline 43 & $396.0-405.5$ & 397.2 & 81.0 & & 7.7 & 75.6 & & - & - & - & - & 16.7 \\
\hline 47 & $434.0-443.5$ & 435.5 & 93.4 & & 12.6 & 87.4 & & - & - & - & - & - \\
\hline 52 & $548.0-557.5$ & 549.3 & 68.7 & & 3.9 & 73.1 & & - & - & - & - & 23.0 \\
\hline \multicolumn{13}{|c|}{$<2 \mu \mathrm{m}$ Fraction } \\
\hline 1 & $0.0-6.5$ & 2.5 & 57.4 & & 25.5 & 12.0 & - & 44.7 & 8.5 & 9.3 & - & - \\
\hline 2 & $6.5-16.0$ & 14.2 & 57.0 & & 22.6 & 7.6 & - & 45.7 & 5.9 & 18.2 & - & - \\
\hline 3 & $16.0-25.5$ & 19.2 & 69.0 & & 25.7 & 10.0 & - & 23.4 & 4.0 & 37.0 & - & - \\
\hline 9 & $73.0-82.5$ & 78.9 & 62.8 & & 23.5 & 11.7 & - & 45.3 & 6.6 & 12.9 & - & - \\
\hline 19 & $168.0-177.5$ & 170.3 & 61.9 & & 21.2 & 9.2 & - & 37.1 & 5.9 & 26.6 & - & - \\
\hline 22 & $196.5-206.0$ & 198.8 & 67.1 & & 28.1 & 11.1 & - & 28.4 & 6.1 & 26.3 & - & - \\
\hline 23 & $206.0-215.5$ & 208.7 & 70.3 & & 33.1 & 12.1 & 5.4 & 21.6 & 2.2 & 25.5 & - & - \\
\hline 26 & $234.5-244.0$ & 239.7 & 65.3 & & 30.5 & 13.0 & 2.2 & 29.0 & 4.9 & 20.4 & - & - \\
\hline 30 & $272.5-282.0$ & 277.7 & 74.0 & & 24.8 & 10.3 & - & 20.6 & 5.0 & 39.3 & - & - \\
\hline 31 & $282.0-291.5$ & 288.1 & 68.1 & & 24.4 & 11.5 & 3.1 & 22.8 & 2.6 & 35.5 & - & - \\
\hline
\end{tabular}


TABLE 10 - Continued

\begin{tabular}{|c|c|c|c|c|c|c|c|c|c|c|c|c|}
\hline Core & $\begin{array}{c}\text { Cored } \\
\text { Interval } \\
\text { Below Sea } \\
\text { Floor (m) }\end{array}$ & $\begin{array}{c}\text { Sample } \\
\text { Below Sea } \\
\text { Floor }(\mathrm{m})\end{array}$ & 客 & गु่ & 㟔 & 远 & $\begin{array}{l}\text { लें } \\
\text { ت্ }\end{array}$ & $\stackrel{\text { J }}{2}$ & 욜 & 茫 & 㤩 & 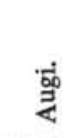 \\
\hline 34 & $310.5-320.0$ & 311.5 & 68.5 & & 28.8 & 14.7 & 2.1 & 17.4 & 2.2 & 34.9 & - & - \\
\hline 36 & $329.5-339.0$ & 331.9 & 67.6 & & 23.8 & 14.8 & 2.6 & 12.5 & 0.9 & 45.4 & - & - \\
\hline \multirow[t]{4}{*}{40} & $367.5-377.0$ & 370.9 & 66.9 & & 1.8 & 38.9 & - & - & 3.9 & 39.1 & 2.6 & 13.8 \\
\hline & & 371.0 & 66.1 & & 3.1 & 42.7 & - & - & - & 37.2 & - & 17.0 \\
\hline & & 372.2 & 69.1 & & 2.2 & 28.5 & - & - & 9.3 & 51.1 & - & 8.8 \\
\hline & & 373.5 & 66.3 & & 3.8 & 43.5 & - & - & - & 42.2 & - & 10.5 \\
\hline \multirow[t]{4}{*}{41} & $377.0-386.5$ & 377.1 & 68.7 & & 2.1 & 50.4 & - & - & - & 34.1 & - & 13.4 \\
\hline & & 378.7 & 67.2 & & 1.4 & 19.3 & - & - & 4.0 & 70.5 & - & 4.8 \\
\hline & & 379.4 & 72.6 & & 2.8 & 61.9 & - & - & - & 24.4 & - & 10.9 \\
\hline & & 380.3 & 69.2 & & 3.1 & 54.3 & - & - & 1.7 & 41.0 & - & - \\
\hline 42 & $386.5-396.0$ & 387.0 & 75.9 & & 3.1 & 69.2 & - & - & 2.4 & - & - & 25.3 \\
\hline 43 & $396.0-405.5$ & 397.2 & 73.0 & & 4.7 & 41.4 & - & - & 1.9 & 42.3 & 1.8 & 8.0 \\
\hline 47 & $434.0-443.5$ & 435.5 & 80.2 & & 4.9 & 36.3 & - & - & 1.5 & 57.4 & - & - \\
\hline 52 & $548.0-557.5$ & 549.3 & 65.0 & & 3.3 & 62.3 & - & - & - & 11.7 & - & 22.6 \\
\hline
\end{tabular}

TABLE 11

Results of X-Ray Diffraction Analysis From Hole 297

\begin{tabular}{|c|c|c|c|c|c|c|c|c|c|c|}
\hline Core & $\begin{array}{l}\text { Cored } \\
\text { Interval } \\
\text { Below Sea } \\
\text { Floor (m) }\end{array}$ & $\begin{array}{l}\text { Sample Depth } \\
\text { Below Sea } \\
\text { Floor (m) }\end{array}$ & 官 & లే & 己ّ & $\frac{0}{a}$ & $\stackrel{\text { J }}{\sum}$ & 일 & हี่ & $\dot{\vec{L}}$ \\
\hline \multicolumn{11}{|c|}{ Bulk Samples } \\
\hline 3 & $20.0-29.5$ & 21.1 & 64.9 & - & 41.9 & 22.1 & 28.5 & 5.7 & 1.8 & \\
\hline 4 & $39.0-48.5$ & 42.8 & 59.5 & - & 40.7 & 18.8 & 32.5 & 5.2 & 2.8 & \\
\hline 5 & $58.0-67.5$ & 64.4 & 54.3 & 18.6 & 33.0 & 16.3 & 25.4 & 3.7 & 3.1 & \\
\hline 6 & $77.0-86.5$ & 83.8 & 61.8 & 10.9 & 34.8 & 20.5 & 25.8 & 4.2 & 3.8 & \\
\hline 12 & $248.0-257.5$ & 250.2 & 61.1 & - & 36.6 & 19.6 & 32.9 & 5.5 & 5.4 & \\
\hline 14 & $314.5-324.0$ & 319.7 & 57.2 & _- & 33.4 & 15.6 & 34.0 & 4.9 & 12.1 & \\
\hline 24 & $618.5-628.0$ & 622.3 & 73.3 & - & 28.6 & 12.8 & 19.2 & 1.7 & 37.7 & \\
\hline 26 & $666.0-675.5$ & 668.8 & 52.8 & - & 34.0 & 13.6 & 28.3 & 1.3 & 22.8 & \\
\hline \multicolumn{11}{|c|}{$2-20 \mu \mathrm{m}$ Fraction } \\
\hline 3 & $20.0-29.5$ & 21.1 & 49.3 & - & 48.1 & 28.6 & 18.6 & 4.7 & - & \\
\hline 4 & $39.0-48.5$ & 42.8 & 50.6 & - & 49.0 & 26.5 & 19.4 & 5.1 & - & \\
\hline 5 & $58.0-67.5$ & 64.4 & 45.7 & - & 46.2 & 27.9 & 20.9 & 4.9 & - & \\
\hline 6 & $77.0-86.5$ & 83.8 & 53.0 & - & 48.1 & 29.0 & 18.3 & 4.6 & - & \\
\hline 12 & $248.0-257.5$ & 250.2 & 50.2 & - & 44.8 & 22.6 & 27.1 & 5.5 & - & \\
\hline 14 & $314.5-324.0$ & 319.7 & 46.5 & - & 49.1 & 23.8 & 23.1 & 4.0 & - & \\
\hline 24 & $618.5-628.0$ & 622.3 & 73.8 & - & 44.9 & 31.0 & 16.3 & 3.0 & 4.9 & \\
\hline 26 & $666.0-675.5$ & 668.8 & 47.6 & - & 49.3 & 23.3 & 25.8 & 1.7 & - & \\
\hline \multicolumn{11}{|c|}{$<2 \mu$ m Fraction } \\
\hline 3 & $20.0-29.5$ & 21.1 & 60.4 & - & 26.1 & 12.5 & 42.4 & 6.7 & 12.4 & - \\
\hline 4 & $39.0-48.5$ & 42.8 & 60.2 & - & 25.7 & 10.8 & 42.6 & 5.9 & 12.2 & 2.7 \\
\hline 5 & $58.0-67.5$ & 64.4 & 54.8 & - & 25.2 & 10.4 & 41.9 & 5.9 & 16.6 & - \\
\hline 6 & $77.0-86.5$ & 83.8 & 56.6 & - & 29.5 & 13.6 & 38.9 & 5.2 & 12.8 & - \\
\hline 12 & $248.0-257.5$ & 250.2 & 60.6 & - & 25.7 & 11.4 & 40.8 & 5.8 & 16.3 & - \\
\hline 14 & $314.5-324.0$ & 319.7 & 64.0 & - & 33.8 & 10.4 & 25.2 & 6.1 & 24.4 & - \\
\hline 24 & $618.5-628.0$ & 622.3 & 55.3 & - & 20.2 & 7.7 & 24.6 & 2.5 & 45.1 & - \\
\hline 26 & $666.0-675.5$ & 668.8 & 58.1 & - & 23.6 & 4.6 & 14.0 & 1.0 & 56.8 & - \\
\hline
\end{tabular}


TABLE 12

Results of X-Ray Diffraction Analysis From Hole 298

\begin{tabular}{|c|c|c|c|c|c|c|c|c|c|c|c|}
\hline Core & $\begin{array}{c}\text { Cored } \\
\text { Interval } \\
\text { Below Sea } \\
\text { Floor }(\mathrm{m})\end{array}$ & $\begin{array}{l}\text { Sample Depth } \\
\text { Below Sea } \\
\text { Floor }(\mathrm{m})\end{array}$ & 定 & $\frac{\dot{J}}{\tilde{J}}$ & 㟧 & $\frac{\vec{\sigma}}{a}$ & $\stackrel{\text { อ̆ }}{\Sigma}$ & $\frac{\dot{0}}{\tilde{U}}$ & ह் & 竞 & 宝 \\
\hline \multicolumn{12}{|c|}{ Bulk Samples } \\
\hline 2 & $126.5-136.0$ & 130.7 & 50.0 & - & 39.5 & 24.9 & 25.4 & 5.4 & 3.7 & 1.1 & \\
\hline 5 & $193.0-202.5$ & 195.8 & 55.9 & 5.6 & 46.8 & 28.3 & 16.2 & 3.0 & - & - & \\
\hline 6 & $278.5-288.0$ & 281.0 & 54.0 & - & 40.7 & 28.3 & 22.7 & 4.2 & 4.2 & - & \\
\hline 9 & $335.5-345.0$ & 336.8 & 56.6 & 3.6 & 31.0 & 24.0 & 30.1 & 6.9 & 3.4 & 1.1 & \\
\hline 11 & $392.5-402.0$ & 395.6 & 50.9 & - & 38.0 & 25.6 & 27.0 & 6.3 & 3.1 & - & \\
\hline 14 & $516.0-525.5$ & 518.6 & 48.8 & 1.8 & 36.6 & 21.8 & 30.5 & 5.4 & 3.9 & - & \\
\hline 15 & $563.5-573.0$ & 570.3 & 51.9 & - & 38.7 & 21.1 & 29.6 & 6,6 & 4.0 & - & \\
\hline 16 & $601.5-611.0$ & 604.3 & 48.5 & - & 39.9 & 23.1 & 24.6 & 5.7 & 5.5 & 1.2 & \\
\hline \multicolumn{12}{|c|}{ 2-20 $\mu \mathrm{m}$ Fraction } \\
\hline 2 & $126.5-136.0$ & 130.7 & 48.1 & - & 48.7 & 30.3 & 16.0 & 4.0 & - & 1.0 & \\
\hline 5 & $193.0-202.5$ & 195.8 & 56.1 & - & 51.5 & 28.7 & 14.9 & 4.9 & - & - & \\
\hline 6 & $278.5-288.0$ & 281.0 & 51.5 & - & 46.4 & 33.4 & 15.4 & 4.8 & - & - & \\
\hline 9 & $335.5-345.0$ & 336.8 & 54.6 & - & 43.3 & 32.3 & 17.9 & 5.4 & - & 1.1 & \\
\hline 11 & $392.5-402.0$ & 395.6 & 49.6 & - & 45.2 & 30.6 & 17.8 & 5.3 & - & 1.1 & \\
\hline 14 & $516.0-525.5$ & 518.6 & 50.6 & - & 47.9 & 30.9 & 14.8 & 5.1 & - & 1.3 & \\
\hline 15 & $563.5-573.0$ & 570.3 & 53.1 & - & 45.8 & 29.1 & 18.4 & 5.6 & - & 1.1 & \\
\hline 16 & $601.5-611.0$ & 604.3 & 51.9 & - & 48.3 & 31.4 & 15.8 & 4.5 & - & - & \\
\hline \multicolumn{12}{|c|}{$<2 \mu$ m Fraction } \\
\hline 2 & $126.5-136.0$ & 130.7 & 63.1 & - & 30.0 & 12.9 & 30.8 & 5.2 & 20.0 & - & 1.1 \\
\hline 5 & $193.0-202.5$ & 195.8 & 57.8 & - & 27.1 & 11.6 & 39.4 & 8.0 & 13.9 & - & - \\
\hline 6 & $278.5-288.0$ & 281.0 & 52.0 & - & 30.6 & 15.8 & 34.9 & 7.5 & 11.3 & - & - \\
\hline 9 & $335.5-345.0$ & 336.8 & 58.6 & - & 29.6 & 13.9 & 38.7 & 8.3 & 9.5 & - & - \\
\hline 11 & $392.5-402.0$ & 395.6 & 50.4 & - & 28.0 & 14.6 & 38.4 & 7.5 & 11.4 & - & - \\
\hline 14 & $516.0-525.5$ & 518.6 & 54.1 & - & 28.0 & 14.6 & 36.9 & 7.6 & 12.9 & - & - \\
\hline 15 & $563.5-573.0$ & 570.3 & 59.4 & - & 30.5 & 14.9 & 37.2 & 6.7 & 10.7 & - & - \\
\hline 16 & $601.5-611.0$ & 604.3 & 55.7 & - & 29.1 & 14.5 & 33.5 & 6.4 & 16.4 & - & - \\
\hline
\end{tabular}

TABLE 13

Results of X-Ray Diffraction Analysis From Hole 298A

\begin{tabular}{|c|c|c|c|c|c|c|c|c|}
\hline Core & $\begin{array}{c}\text { Cored } \\
\text { Interval } \\
\text { Below Sea } \\
\text { Floor }(\mathrm{m})\end{array}$ & $\begin{array}{c}\text { Sample Depth } \\
\text { Below Sea } \\
\text { Floor }(\mathrm{m})\end{array}$ & 岁 & 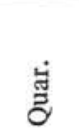 & $\frac{\dot{m}}{20}$ & ¿ूల & 롱 & $\stackrel{\dot{\Xi}}{\stackrel{0}{\Sigma}}$ \\
\hline \multicolumn{9}{|c|}{ Bulk Samples } \\
\hline 1 & $50.5-60.0$ & 51.9 & 52.5 & 43.6 & 24.7 & 22.3 & 4.8 & 4.6 \\
\hline \multicolumn{9}{|c|}{$2-20 \mu \mathrm{m}$ Fraction } \\
\hline 1 & $50.5-60.0$ & 51.9 & 51.0 & 50.5 & 31.3 & 13.9 & 4.3 & - \\
\hline \multicolumn{9}{|c|}{$<2 \mu$ m Fraction } \\
\hline 1 & $50.5-60.0$ & 51.9 & 49.4 & 27.6 & 13.4 & 39.5 & 7.5 & 12.0 \\
\hline
\end{tabular}


TABLE 14

Results of X-Ray Diffraction Analysis From Hole 299

\begin{tabular}{|c|c|c|c|c|c|c|c|c|c|c|c|c|c|c|}
\hline Core & $\begin{array}{c}\text { Cored } \\
\text { Interval } \\
\text { Below Sea } \\
\text { Floor }(\mathrm{m})\end{array}$ & $\begin{array}{l}\text { Sample Depth } \\
\text { Below Sea } \\
\text { Floor (m) }\end{array}$ & 总 & ن் & $\dot{\circ}$ & 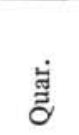 & 它 & $\frac{a}{2}$ & 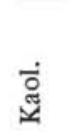 & 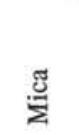 & 열 & 茂 & ఏ & 家 \\
\hline \multicolumn{15}{|c|}{ Bulk Samples } \\
\hline $\begin{array}{r}9 \\
18 \\
26 \\
33 \\
38\end{array}$ & $\begin{array}{c}47.5-57.0 \\
\\
76.0-85.5 \\
161.5-171.0 \\
237.5-247.0 \\
418.0-427.5 \\
522.5-532.0\end{array}$ & $\begin{array}{r}54.5 \\
54.7 \\
82.5 \\
166.9 \\
238.5 \\
420.4 \\
523.6\end{array}$ & $\begin{array}{l}59.4 \\
70.2 \\
69.9 \\
67.7 \\
62.3 \\
52.9 \\
53.1\end{array}$ & $\begin{array}{l}- \\
5.7 \\
3.8 \\
- \\
- \\
- \\
-\end{array}$ & $\begin{array}{l}1.8 \\
- \\
- \\
- \\
- \\
- \\
-\end{array}$ & $\begin{array}{l}41.4 \\
32.5 \\
30.5 \\
39.3 \\
35.7 \\
30.8 \\
24.6\end{array}$ & $\begin{array}{l}3.4 \\
4.9 \\
4.6 \\
5.8 \\
8.6 \\
3.8 \\
3.7\end{array}$ & $\begin{array}{l}19.9 \\
16.9 \\
26.2 \\
25.7 \\
23.9 \\
17.1 \\
14.3\end{array}$ & $\begin{array}{l}- \\
- \\
3.5 \\
- \\
- \\
-\end{array}$ & $\begin{array}{l}23.9 \\
24.7 \\
15.0 \\
15.6 \\
13.8 \\
25.2 \\
16.5\end{array}$ & $\begin{array}{l}5.6 \\
4.2 \\
2.6 \\
2.1 \\
2.1 \\
5.6 \\
5.1\end{array}$ & \begin{tabular}{r|}
2.3 \\
3.2 \\
10.3 \\
11.4 \\
12.9 \\
16.2 \\
30.3
\end{tabular} & $\begin{array}{l}- \\
- \\
- \\
- \\
- \\
2.7\end{array}$ & $\begin{array}{l}1.7 \\
7.8 \\
3.5 \\
- \\
3.0 \\
1.2 \\
2.7\end{array}$ \\
\hline \multicolumn{15}{|c|}{$2-20 \mu \mathrm{m}$ Fraction } \\
\hline $\begin{array}{r}9 \\
18 \\
26 \\
33 \\
38\end{array}$ & $\begin{array}{c}47.5-57.0 \\
76.0-85.5 \\
161.5-171.0 \\
237.5-247.0 \\
418.0-427.5 \\
522.5-532.0\end{array}$ & $\begin{array}{r}54.5 \\
54.7 \\
82.5 \\
166.9 \\
238.5 \\
420.4 \\
523.6\end{array}$ & $\begin{array}{l}44.6 \\
63.1 \\
65.9 \\
67.8 \\
67.6 \\
59.9 \\
56.3\end{array}$ & $\begin{array}{l}- \\
- \\
- \\
- \\
- \\
-\end{array}$ & $\begin{array}{l}- \\
- \\
- \\
- \\
- \\
-\end{array}$ & $\begin{array}{l}41.4 \\
40.2 \\
33.8 \\
38.7 \\
40.8 \\
39.9 \\
33.5\end{array}$ & $\begin{array}{r}4.6 \\
5.2 \\
12.2 \\
13.2 \\
9.8 \\
- \\
4.8\end{array}$ & $\begin{array}{l}22.6 \\
26.7 \\
34.7 \\
34.4 \\
33.5 \\
22.3 \\
21.5\end{array}$ & $\begin{array}{l}- \\
- \\
3.1 \\
- \\
- \\
- \\
-\end{array}$ & $\begin{array}{r}23.4 \\
14.2 \\
8.8 \\
11.5 \\
9.8 \\
25.0 \\
16.0\end{array}$ & $\begin{array}{l}5.5 \\
3.6 \\
1.9 \\
2.1 \\
2.3 \\
6.0 \\
5.7\end{array}$ & $\begin{array}{c}- \\
- \\
- \\
- \\
\overline{5} .3 \\
10.2\end{array}$ & $\begin{array}{l}- \\
- \\
- \\
- \\
- \\
1.6 \\
4.2\end{array}$ & $\begin{array}{r}2.6 \\
10.1 \\
5.7 \\
\overline{3} .7 \\
\overline{4} .0\end{array}$ \\
\hline \multicolumn{15}{|c|}{$<2 \mu$ Fraction } \\
\hline $\begin{array}{r}9 \\
18 \\
26 \\
33 \\
38\end{array}$ & $\begin{array}{c}47.5-57.0 \\
\\
76.0-85.5 \\
161.5-171.0 \\
237.5-247.0 \\
418.0-427.5 \\
522.5-532.0\end{array}$ & $\begin{array}{r}54.5 \\
54.7 \\
82.5 \\
166.9 \\
238.5 \\
420.4 \\
523.6\end{array}$ & $\begin{array}{l}58.6 \\
69.2 \\
67.1 \\
71.1 \\
69.4 \\
56.5 \\
56.9\end{array}$ & $\begin{array}{l}- \\
- \\
- \\
- \\
- \\
-\end{array}$ & $\begin{array}{l}- \\
- \\
- \\
- \\
- \\
-\end{array}$ & $\begin{array}{l}24.5 \\
26.9 \\
20.0 \\
22.7 \\
26.1 \\
22.9 \\
22.5\end{array}$ & $\begin{array}{l}3.6 \\
4.1 \\
6.5 \\
6.4 \\
5.7 \\
2.3 \\
3.6\end{array}$ & $\begin{array}{l}11.9 \\
14.1 \\
14.0 \\
12.3 \\
12.4 \\
10.2 \\
11.8\end{array}$ & $\begin{array}{l}- \\
- \\
6.1 \\
- \\
- \\
-\end{array}$ & $\begin{array}{l}42.1 \\
30.3 \\
18.4 \\
22.5 \\
22.3 \\
30.3 \\
15.8\end{array}$ & $\begin{array}{l}6.4 \\
4.7 \\
4.0 \\
4.3 \\
4.0 \\
5.4 \\
4.4\end{array}$ & $\begin{array}{l}11.4 \\
13.2 \\
27.5 \\
30.5 \\
26.9 \\
28.8 \\
41.8\end{array}$ & $\begin{array}{l}- \\
- \\
1.3 \\
- \\
- \\
-\end{array}$ & $\begin{array}{l}\overline{6} .6 \\
3.4 \\
\overline{2} .5 \\
- \\
-\end{array}$ \\
\hline
\end{tabular}


TABLE 15

Results of X-Ray Diffraction Analysis From Hole 301

\begin{tabular}{|c|c|c|c|c|c|c|c|c|c|c|c|c|c|c|}
\hline Core & $\begin{array}{c}\text { Cored } \\
\text { Interval } \\
\text { Below Sea } \\
\text { Floor }(\mathrm{m})\end{array}$ & $\begin{array}{l}\text { Sample Depth } \\
\text { Sea Below } \\
\text { Floor }(\mathrm{m})\end{array}$ & 宅 & गुँ & 융 & 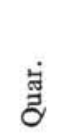 & 荘 & 孪 & $\begin{array}{l}\dot{0} \\
\text { đ }\end{array}$ & 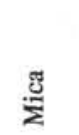 & 을 & 莣 & 离 & 竞 \\
\hline \multicolumn{15}{|c|}{ Bulk Samples } \\
\hline 2 & $117.0-126.5$ & 120.7 & 61.4 & 2.0 & - & 36.0 & 6.0 & 19.7 & - & 24.8 & 3.9 & 1.9 & 5.9 & - \\
\hline \multirow[t]{2}{*}{4} & $155.0-164.5$ & 157.3 & 61.2 & - & - & 33.7 & 4.0 & 21.8 & 1.1 & 27.3 & 4.1 & 6.6 & 1.5 & - \\
\hline & & 157.7 & 67.8 & - & - & 30.5 & 5.9 & 19.5 & 3.1 & 24.7 & 3.1 & 10.7 & 2.4 & - \\
\hline 8 & $240.5-250.0$ & 246.0 & 78.7 & - & - & 37.3 & 8.1 & 15.0 & 1.0 & 28.0 & 3.9 & 4.1 & 2.7 & - \\
\hline 13 & $354.5-364.0$ & 355.8 & 69.7 & - & - & 39.0 & 7.5 & 18.6 & - & 18.9 & 2.7 & 10.2 & 3.1 & - \\
\hline 15 & $392.5-402.0$ & 395.4 & 42.4 & - & 1.7 & 37.8 & 15.7 & 27.1 & - & 13.4 & 0.6 & - & 1.8 & 1.8 \\
\hline 17 & $449.5-459.0$ & 450.3 & 48.4 & - & - & 37.0 & 16.9 & 27.7 & - & 11.1 & 1.0 & 5.3 & 1.0 & - \\
\hline \multirow[t]{2}{*}{18} & $468.5-478.0$ & 474.2 & 65.9 & - & - & 37.5 & 5.6 & 18.2 & - & 20.8 & 2.5 & 13.7 & 1.7 & - \\
\hline & & 474.4 & 67.7 & - & - & 29.0 & 5.8 & 20.4 & - & 18.8 & 3.8 & 18.5 & 3.6 & - \\
\hline \multicolumn{15}{|c|}{ 2-20 $\mu \mathrm{m}$ Fraction } \\
\hline 2 & $117.0-126.5$ & 120.7 & 54.3 & - & - & 42.7 & 7.1 & 26.5 & - & 16.2 & 2.9 & - & 4.6 & - \\
\hline \multirow[t]{2}{*}{4} & $155.0-164.5$ & 157.3 & 56.6 & - & - & 40.5 & 7.6 & 28.9 & - & 17.7 & 3.3 & - & 2.0 & - \\
\hline & & 157.7 & 58.2 & - & - & 36.8 & 5.7 & 26.2 & - & 23.2 & 3.9 & - & 4.2 & - \\
\hline 8 & $240.5-250.0$ & 246.0 & 70.7 & - & - & 44.1 & 6.7 & 20.2 & - & 21.5 & 2.8 & - & 4.6 & - \\
\hline 13 & $354.5-364.0$ & 355.8 & 68.5 & - & - & 47.4 & 8.0 & 23.7 & _- & 14.2 & 2.6 & - & 4.2 & - \\
\hline 15 & $392.5-402.0$ & 395.4 & 56.8 & - & - & 36.0 & 10.1 & 31.7 & - & 14.9 & 0.8 & - & 3.7 & 2.8 \\
\hline 17 & $449.5-459.0$ & 450.3 & 54.4 & - & - & 34.9 & 8.3 & 29.4 & - & 19.4 & 2.5 & - & 5.5 & - \\
\hline \multirow{2}{*}{18} & $468.5-478.0$ & 474.2 & 65.5 & - & - & 43.4 & 6.6 & 23.7 & - & 19.1 & 4.7 & - & 2.6 & - \\
\hline & & 474.4 & 65.1 & - & - & 33.3 & 6.0 & 28.2 & - & 17.8 & 3.8 & 7.3 & 3.6 & - \\
\hline \multicolumn{15}{|c|}{$<2 \mu \mathrm{m}$ Fraction } \\
\hline 2 & $117.0-126.5$ & 120.7 & 63.3 & - & - & 24.3 & 4.7 & 12.1 & - & 32.8 & 7.0 & 12.9 & 6.1 & - \\
\hline \multirow[t]{2}{*}{4} & $155.0-164.5$ & 157.3 & 65.9 & - & - & 21.6 & 3.9 & 12.6 & 3.5 & 19.3 & 2.4 & 36.8 & - & - \\
\hline & & 157.7 & 68.6 & _- & - & 22.4 & 4.9 & 12.9 & 5.3 & 24.9 & 2.5 & 25.6 & 1.4 & - \\
\hline 8 & $240.5-250.0$ & 246.0 & 79.5 & - & - & 24.4 & 5.1 & 11.3 & 2.5 & 38.3 & 5.2 & 11.7 & 1.4 & - \\
\hline 13 & $354.5-364.0$ & 355.8 & 78.4 & - & - & 26.1 & 4.2 & 12.3 & 3.1 & 25.2 & 3.1 & 22.7 & 3.4 & - \\
\hline 15 & $392.5-402.0$ & 395.4 & 66.4 & - & - & 15.2 & 3.9 & 9.9 & 5.1 & 30.0 & 4.1 & 27.9 & 3.8 & - \\
\hline 17 & $449.5-459.0$ & 450.3 & 62.7 & - & - & 16.0 & 3.7 & 9.7 & 3.3 & 27.0 & 4.2 & 34.9 & 1.2 & - \\
\hline \multirow[t]{2}{*}{18} & $468.5-478.0$ & 474.2 & 69.7 & - & - & 24.4 & 3.0 & 12.4 & 3.4 & 25.3 & 2.9 & 27.5 & 1.1 & - \\
\hline & & 474.4 & 67.0 & - & - & 16.2 & 2.4 & 8.6 & 1.8 & 12.0 & 2.8 & 54.3 & 1.8 & - \\
\hline
\end{tabular}


TABLE 16

Results of X-Ray Diffraction Analysis From Hole 302

\begin{tabular}{|c|c|c|c|c|c|c|c|c|c|c|c|c|c|c|}
\hline Core & $\begin{array}{c}\text { Cored } \\
\text { Interval } \\
\text { Below Sea } \\
\text { Floor }(\mathrm{m})\end{array}$ & $\begin{array}{l}\text { Sample Depth } \\
\text { Below Sea } \\
\text { Floor }(\mathrm{m})\end{array}$ & 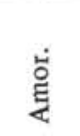 & 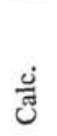 & 芯 & $\dot{m}$ & 这 & $\frac{\ddot{m}}{2}$ & $\begin{array}{l}\overrightarrow{0} \\
\ddot{\ddot{I}}\end{array}$ & $\frac{3}{2}$ & $\dot{\mathcal{E}}$ & $\vec{E}$ & 胥 & 崖 \\
\hline \multicolumn{15}{|c|}{ Bulk Samples } \\
\hline 2 & $19.0-28.5$ & 26.3 & 62.8 & 2.9 & 41.2 & - & - & 18.8 & & 28.7 & 4.0 & 3.2 & - & 1.1 \\
\hline 3 & $38.0-47.5$ & 38.7 & 65.7 & - & 32.3 & - & 4.6 & 18.6 & & 29.2 & 3.9 & 11.4 & - & - \\
\hline 5 & $76.0-85.5$ & 82.0 & 82.3 & - & 37.6 & - & 4.1 & 17.3 & & 28.4 & 4.4 & 6.3 & - & 1.9 \\
\hline 7 & $114.0-123.5$ & 120.9 & 79.0 & - & 36.4 & - & 3.9 & 17.1 & & 29.2 & 4.2 & 9.2 & - & - \\
\hline 10 & $171.0-180.5$ & 171.6 & 80.4 & - & 31.7 & - & 7.2 & 16.0 & & 26.7 & 3.0 & 10.5 & - & 4.9 \\
\hline 15 & $266.0-275.5$ & 266.9 & 94.4 & - & 33.7 & - & 7.2 & 16.4 & & 8.0 & 3.3 & 20.1 & - & 11.2 \\
\hline 17 & $456.0-465.5$ & 456.1 & 50.6 & - & 19.9 & 32.7 & 3.0 & 7.5 & & 13.0 & 1.3 & 17.0 & 1.4 & 4.2 \\
\hline \multicolumn{15}{|c|}{$2-20 \mu \mathrm{m}$ Fraction } \\
\hline 2 & $19.0-28.5$ & 26.3 & 48.9 & - & 45.5 & - & 8.4 & 24.3 & & 18.3 & 3.5 & - & - & - \\
\hline 3 & $38.0-47.5$ & 38.7 & 50.7 & - & 42.5 & - & 7.8 & 23.1 & & 21.1 & 4.4 & - & - & 1.0 \\
\hline 5 & $76.0-85.5$ & 82.0 & 79.4 & - & 52.7 & - & 5.8 & 25.4 & & 12.9 & 2.1 & - & - & 1.2 \\
\hline 7 & $114.0-123.5$ & 120.9 & 78.8 & - & 47.7 & - & 5.7 & 22.4 & & 20.0 & 4.1 & - & - & - \\
\hline 10 & $171.0-180.5$ & 171.6 & 71.6 & - & 42.0 & - & 5.2 & 23.0 & & 21.8 & 5.2 & - & - & 2.7 \\
\hline 15 & $266.0-275.5$ & 266.9 & 94.5 & - & 39.8 & - & 9.2 & 22.2 & & 15.8 & 1.6 & - & - & 11.4 \\
\hline 17 & $456.0-465.5$ & 456.1 & 28.8 & - & 29.5 & 25.2 & 4.0 & 13.3 & & 18.7 & 2.6 & - & 1.2 & 5.6 \\
\hline \multicolumn{15}{|c|}{$<2 \mu \mathrm{m}$ Fraction } \\
\hline 2 & $19.0-28.5$ & 26.3 & 62.1 & - & 28.1 & - & 5.4 & 13.8 & 3.3 & 37.1 & 4.7 & 7.6 & - & - \\
\hline 3 & $38.0-47.5$ & 38.7 & 70.2 & - & 25.7 & - & 4.3 & 11.7 & - & 27.7 & 4.6 & 22.5 & - & 3.4 \\
\hline 5 & $76.0-85.5$ & 82.0 & 81.5 & - & 30.2 & - & 3.5 & 10.9 & 3.0 & 35.8 & 4.1 & 12.5 & - & - \\
\hline 7 & $114.0-123.5$ & 120.9 & 72.8 & - & 21.2 & - & 2.8 & 10.5 & - & 35.6 & 4.1 & 25.9 & - & - \\
\hline 10 & $171.0-180.5$ & 171.6 & 74.2 & - & 20.9 & - & 3.7 & 8.4 & 1.5 & 29.3 & 2.7 & 33.5 & - & - \\
\hline 15 & $266.0-275.5$ & 266.9 & 90.8 & - & 24.0 & - & 5.2 & 11.7 & - & 16.1 & 2.0 & 36.7 & - & 4.3 \\
\hline 17 & $456.0-465.5$ & 456.1 & 25.7 & - & 7.8 & 53.5 & 1.5 & 1.8 & - & 7.3 & 0.7 & 21.8 & 4.7 & 0.9 \\
\hline
\end{tabular}

